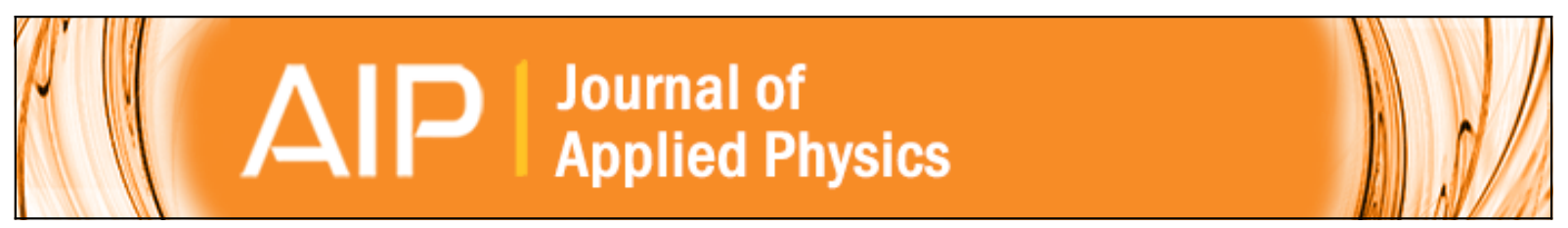

\title{
Study on the dynamics responses of a transmission system made from carbon nanotubes
}

Hang Yin, Kun Cai, Ning Wei, Qing-Hua Qin, and Jiao Shi

Citation: Journal of Applied Physics 117, 234305 (2015); doi: 10.1063/1.4922713

View online: http://dx.doi.org/10.1063/1.4922713

View Table of Contents: http://scitation.aip.org/content/aip/journal/jap/117/23?ver=pdfcov

Published by the AIP Publishing

\section{Articles you may be interested in}

A stable high-speed rotational transmission system based on nanotubes

Appl. Phys. Lett. 106, 021909 (2015); 10.1063/1.4905696

Analysis of a three phase induction motor directly from Maxwell's equations

Am. J. Phys. 80, 43 (2012); 10.1119/1.3633696

Torsional behavior of chiral single-walled carbon nanotubes is loading direction dependent Appl. Phys. Lett. 90, 201910 (2007); 10.1063/1.2739325

A comparative analysis of permanent magnet-type bearingless synchronous motors for fully magnetically levitated rotors

J. Appl. Phys. 83, 7121 (1998); 10.1063/1.367540

The effect of different profiles of the rotor magnet in an aquatic ac synchronous motor

J. Appl. Phys. 81, 4767 (1997); 10.1063/1.365458

\section{Đ SHIMADZU Powerful, Multi-functional UV-Vis-NIR and Excellence in science FTIR Spectrophotometers}

Providing the utmost in sensitivity, accuracy and resolution for applications in materials characterization and nano research

- Photovoltaics - Ceramics

- Polymers - DNA film structures

- Thin films - Coatings

- Paints - Packaging materials
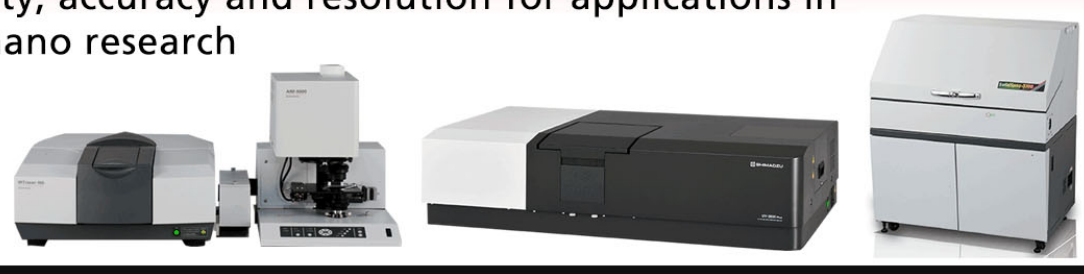


\title{
Study on the dynamics responses of a transmission system made from carbon nanotubes
}

\author{
Hang Yin, ${ }^{1}$ Kun Cai, ${ }^{1, a)}$ Ning Wei, ${ }^{1}$ Qing-Hua Qin, ${ }^{2}$ and Jiao Shi ${ }^{3}$ \\ ${ }^{1}$ College of Water Resources and Architectural Engineering, Northwest A\&F University, \\ Yangling 712100, China \\ ${ }^{2}$ Research School of Engineering, The Australian National University, Canberra, Australian Capital \\ Territory 2601, Australia \\ ${ }^{3}$ Department of Civil and Environmental Engineering, University of Missouri, Columbia, \\ Missouri 65211-2200, USA
}

(Received 26 March 2015; accepted 20 May 2015; published online 16 June 2015)

A rotational transmission system from coaxial carbon nanotubes (CNTs) is investigated using a computational molecular dynamics approach. The system consists of a motor from a single-walled carbon nanotube and a bearing from a double-walled carbon nanotube. The motor has a high fixed rotational frequency and the two ends of the outer tube in the bearing are fixed. The inner tube in the bearing works as a rotor. Because of the interlayer friction in the bearing, configurations of the joint between the adjacent ends of motor and rotor have significant effects on rotational transmission properties. Four factors are considered in simulation, i.e., the bonding types of atoms $\left(s p^{1}\right.$ and $\left.s p^{2}\right)$ on the ends of motor and rotor, the difference between motor and rotor radii, the rotational speed of motor, and the environmental temperature. It is found that the synchronous transmission happens if the $s p^{1}$ atoms on the jointed ends of motor and rotor are bonded each other and become new $s p^{2}$ atoms. Therefore, the lower difference between radii of motor and rotor, higher temperature of environment leads to synchronous rotational transmission easily. If the environmental temperature is too low (e.g., $<150 \mathrm{~K}$ ), the end of motor adjacent to rotor is easily under buckling and new $s p^{2}$ atoms appear, too. With capped CNTs or higher radii difference between rotor and motor at an appropriate temperature, a stable asynchronous rotation of rotor can be generated, and the rotor's frequency varying linearly with motor's frequency between 230 and $270 \mathrm{GHz}$. A multi-signal transmission device combined with oscillating and rotational motion is proposed for motor and stator shares a same size in radius. (C) 2015 AIP Publishing LLC.

[http://dx.doi.org/10.1063/1.4922713]

\section{INTRODUCTION}

In recent decades, carbon nanotubes (CNTs) have attracted extensive attention in a variety of fields of nanoengineering due to their excellent mechanical, electrical, and thermal properties. ${ }^{1-6}$ In particular, they have been considered important candidates as building blocks for the next generation of nano-electro-mechanical systems (NEMS). ${ }^{7}$ In these applications, mechanisms of relative movement (such as axial linear ${ }^{8,9}$ and rotational ${ }^{6,10,11}$ movements) between adjacent walls of a multi-wall CNT (MWCNT) are of importance. These mechanisms have been taken as a fundamental basis for designing nano devices such as gigahertz oscillators $^{2,12-15}$ and motors ${ }^{1,16-18}$ For example, Subramanian et al. ${ }^{19}$ achieved nanoscale batch fabrication of CNT bearings. Fennimore et al. ${ }^{11}$ synthesized a rotational motor with a metal plate motor attached to the outer shells of MWCNTs and activated by three stator electrodes. Soon after that Bourlon et al. ${ }^{10}$ changed the attachment position of the rotational plate to the rotors of MWCNTs. These methods suggested a feasible way to rotate MWCNTs axially, motivating much further study of the rotation of CNTs during the last decade.

\footnotetext{
a) Author to whom correspondence should be addressed. Electronic mail: kuicansj@163.com. Tel.: +86 15102916861.
}

In investigations of the rotational movement of MWCNTs, the bolt and nut pair ${ }^{20-22}$ among adjacent layers is one of the major factors to be considered. Belikov et al. ${ }^{22}$ reported that potential energy barriers with different chirality and helical angles could lead to different screw-like motions. A number of studies ensued from that discovery. For example, Schoen et $a l .{ }^{3}$ simulated golden mass transportation inside CNTs under a thermal gradient, and found that the trajectories were helical. Similarly, the transport of cargoes attached to the outer shells of double-walled carbon nanotubes (DWCNTs) was studied using both experiment and molecular dynamics (MD) simulation methods. ${ }^{23}$ These types of motion can be regarded as CNT-based motors with a shorter CNT moving axially along a longer CNT. ${ }^{17}$ Departing from the thermal gradient condition, Somada et al. ${ }^{1}$ represented linear motors under constant temperature between 1073 and $1373 \mathrm{~K}$. The short-capped CNT moved forward and back constantly and could be regarded as an oscillator. In recent work, we reported self-excited gigahertz oscillations ${ }^{12}$ that were mainly started by the bolt and nut pair and the end effect of CNTs. ${ }^{24}$ Apart from thermal energy, electric energy is another way to trigger CNT motors. Tu and $\mathrm{Hu}^{18}$ designed an electrical motor by applying axially varying voltage under an isothermal condition. By applying a similar condition, Hamdi et al. ${ }^{16}$ assigned two 
opposing MWCNTs in a direct current circuit. By virtue of the ultralow friction force between adjacent walls, the inner shells move out easily and begin to rotate. When the rotors contact each other, rotation is then the only motion for the whole system. Kang and Hwang ${ }^{25}$ simulated a high-speed rotational CNT motor activated by friction between CNT wall surface and fluidic gas. Zhao et al. ${ }^{26}$ proposed the coarse-grained potentials to simulate single-walled carbon nanotube (SWCNT) bundles and buckypaper in high efficiency and high accuracy. Wang and $\mathrm{Arash}^{27}$ reviewed the experimental and simulation works of nano-resonator sensors based on CNTs and graphenes. A series of promising application studies generate a great prospect for designing nano devices with CNTs.

As demonstrated in the above review, the friction between tubes plays a major role in the dynamic systems, e.g., oscillator or rotator. Considering the rotation of CNTs is hard to generate and control, we suggested a feasible way for controlling the output rotational frequency through a stable rotational transmission system (RTS). ${ }^{28}$ For such system, the tube ends were bonded with hydrogen atoms to reduce the strong edge effect. In the present study, we will discuss the effect of tube end configuration, radii differences, and temperature on the rotational transmission behavior. MD simulations are adopted to investigate the dynamic behavior of such system. The results have the potential to provide ways of fabricating some new nano devices.

\section{METHODS}

A system consisting of a coaxial SWCNT and a DWCNT is employed in this work. One is assumed to be a rotational motor and the other is regarded as transmission device. For the force field used in our MD simulation with LAMMPS, ${ }^{29}$ the adaptive intermolecular reactive empirical bond order (AIREBO) potential ${ }^{30}$ is chosen to describe the covalent bonds among carbon atoms and long-range van der Waals interaction force. Quantum-ESPRESSO ${ }^{31}$ is used to find the electron density distribution.

Before conducting the simulation, a Nosé-Hoover heat bath at a constant temperature is applied to relax the whole coaxial system except for 3 fixed layers of tube ends. Except in discussing the temperature effect, all the environmental temperature is $300 \mathrm{~K}$. The duration of the relaxation time is $100 \mathrm{ps}$. After relaxation, the motor tubes begin rotating with a period of $4 \mathrm{ps}$ (rotational frequency $250 \mathrm{GHz}$ ) without translational motion along their axis. The two ends of the outer tube are fixed with 3 layers on each end. At the same time, the rotors of the DWCNTs are set freely. The whole system is under the canonical NVT ensemble ( $\mathrm{N}$ is the total number of particles in the system, $\mathrm{V}$ is the system's volume, and $\mathrm{T}$ is the absolute temperature). For all simulations after relaxation, $5-10 \mathrm{~ns}$ are simulated with the time step of $1 \mathrm{fs}$.

\section{MODELS}

In this work, all tubes are assumed to have armchair chirality. The right part in Fig. 1(a) shows the configuration of a bearing from a DWCNT of $(5,5) /(10,10)$, where $(5,5)$ are the chiral indices of the rotor and $(10,10)$ are those of the outer tube. The lengths of the rotor (blue) and outer tube (orange) are about $6 \mathrm{~nm}$ and $4 \mathrm{~nm}$, respectively. The left part (pink) is a SWCNT-based driving motor with length of $2.1 \mathrm{~nm}$. The initial distance between driving motor and rotor is $0.34 \mathrm{~nm}$, which is the balance distance between two graphite sheets.

\section{(a) BO model}

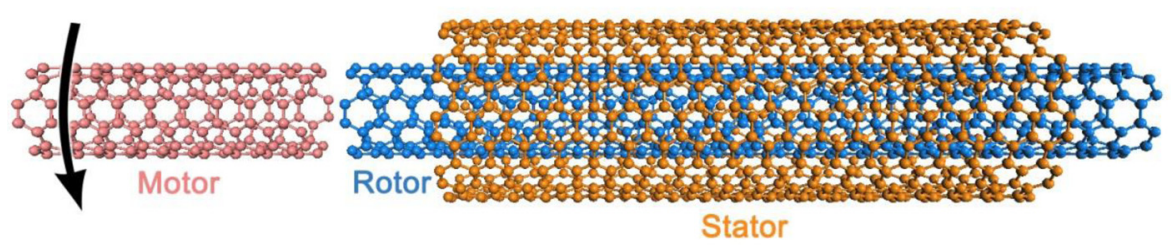

(b) MC model

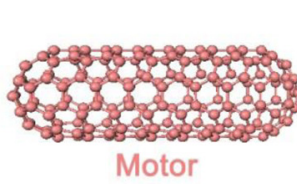

Motor

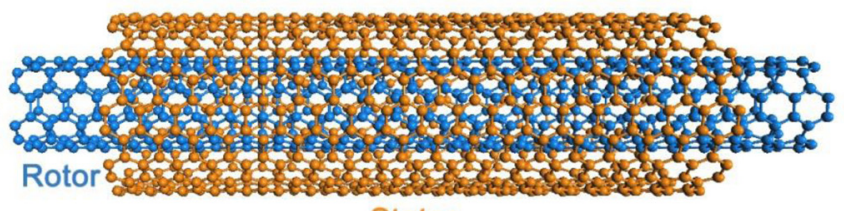

Stator
FIG. 1. Models for asynchronous rotation transmission systems containing a $(5,5)$ driving motor and $(5,5) /(10,10)$ DWCNT. (a) Ends of both motor and rotor are open (BO model), (b) ends of motor are capped (MC model), and (c) both motor and rotor are end capped (BC model).

\section{(c) BC model}
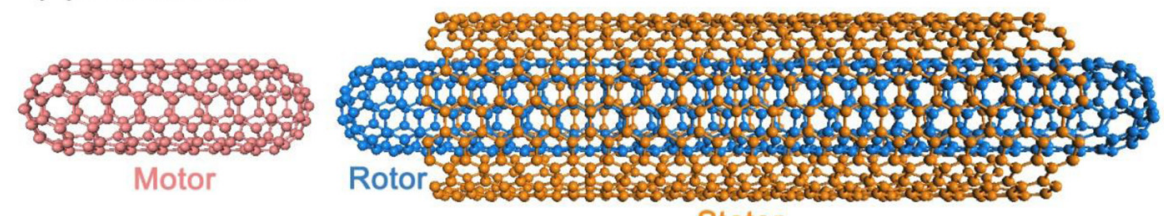

Stator 
Based on the system above, the effects of the configurations of tube ends and the radii of the motor on its dynamic behavior are investigated. In particular, the configurations of the ends of a motor of index $(5,5)$ and rotor can be classified into three types: both open (BO), only motor capped (MC), and both capped (BC) (Fig. 1). The cap of a tube is half of a C60 buckyball. Second, to investigate the effect of the radii of the motor on the dynamic behavior of the bearing, we employ six armchair models of the motor from $(5,5)$ to $(10,10)$.

\section{RESULTS AND DISCUSSION}

\section{A. Effects of tube end configurations}

In a transmission system, when the degree of freedom (DOF) constraint is removed from the rotor, it can move forward to its right end through the interaction force of the tubes' ends. Fig. 3 illustrates the history of rotational frequency and positions of the mass center of inner tubes/rotors (MCITs) of the three configurations shown in Fig. 1. The investigation indicated that the stable position is independent of initial position. The difference between the relative positions of MCIT in Fig. 3 is mainly caused by the size of either one cap (MC) or two caps (BC). After 4000 ps of simulation, stable position is achieved. For the BO condition of tube ends, the carbon structure has $s p^{1}$ orbital hybridization (i.e., $s p^{1}$ bonded carbon atoms) on tube ends (Fig. 1(a)). The remaining $p$ orbit electrons have strong interactions between two adjacent tube ends. Fig. 2(a) shows the electron density

(a) BO

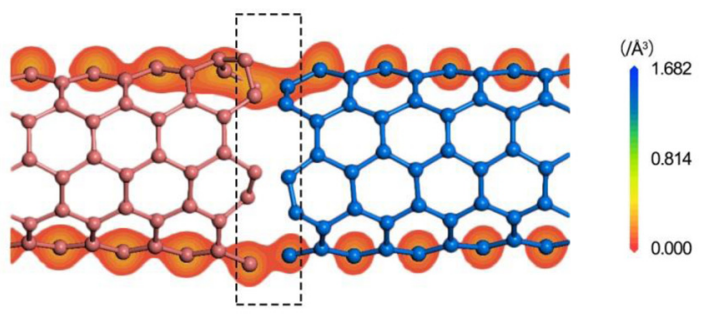

(b) MC
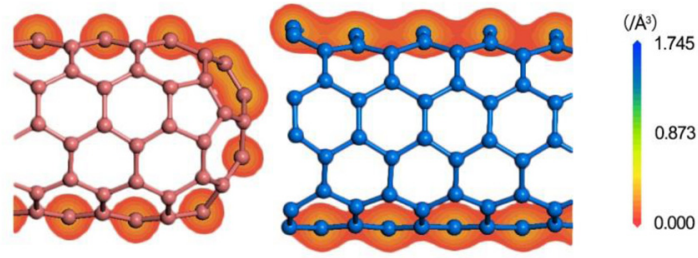

(c) $\mathrm{BC}$
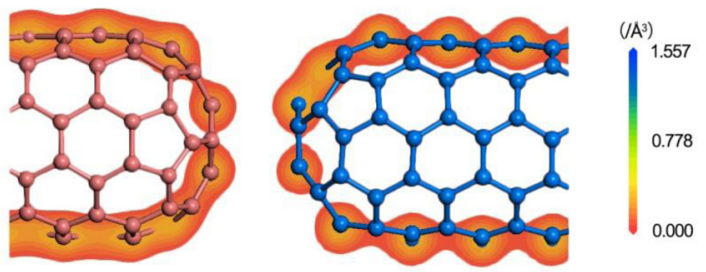

FIG. 2. Electron density distribution in a longitudinal cross section of joint with different types of ends: (a) BO, (b) MC, and (c) BC ends at $1800 \mathrm{ps}$. In (a), $s p^{2}$ bonded atoms appear. In (b) and (c), there is no new $s p^{2}$ bonded atoms. distribution near the joint in the system shown in Fig. 3(a) after relaxation, demonstrating that the interaction is the strongest among the three cases of $\mathrm{BO}, \mathrm{MC}$, and $\mathrm{BC}$. Hence, we conclude that the rotation of the rotor is triggered immediately after the DOF constraint is removed. When both ends are capped, however (BC), there is only interaction among $s p^{2}$ bonded atoms at the ends of motor and rotor, which is directly shown by the electron density distribution near the joint in Fig. 2(c). The interaction is much weaker than that between $s p^{1}$ bonded atoms. Such interaction provides less torque to drive the rotation of the rotor. Asynchronous rotation transmission occurs readily. It should be mentioned that longer initial distance between the motor and rotor may lead to longer delay of the appearing of peak value of rotational frequency of rotor. Smaller overlap of electron densities between two neighbor ends of motor and rotor may result in lower peak value of rotation speed.

If the motor has capped ends and the rotor has open ends (MC), the interaction between $s p^{2}$ and $s p^{1}$ bonded atoms is stronger than that between $s p^{2}$ bonded atoms but weaker than that between $s p^{1}$ bonded atoms.

For the system with both open ends (BO), the rotor's rotational frequency (black line in Fig. 3(a)) varies near $250 \mathrm{GHz}$ (yellow broken line), which is the same value as that of motor's rotation speed. From Fig. 3(b), we find that the position of MCIT (black line) remains almost unchanged, demonstrating that the rotor in the bearing has no translational
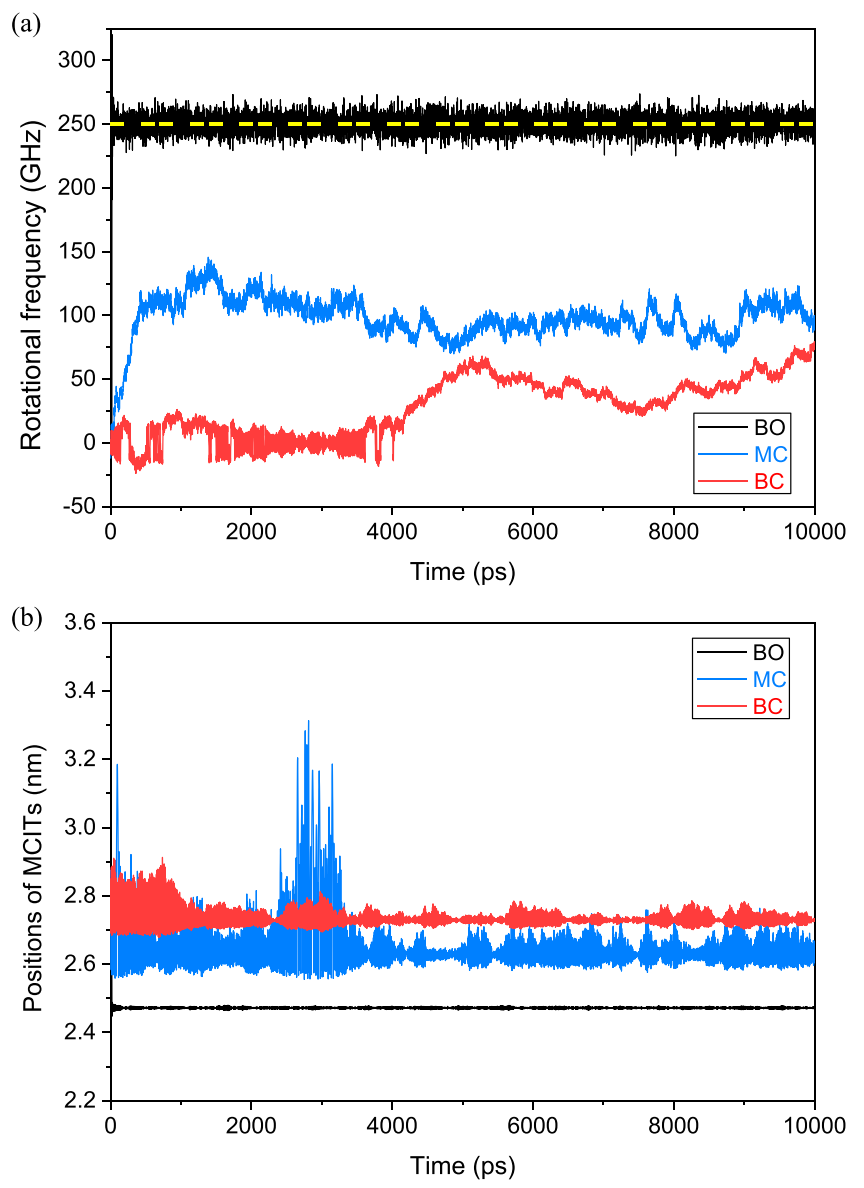

FIG. 3. Histories of rotational frequency and positions of mass center of rotor (MCITs) with different joint configurations. 
motion after being absorbed by the right end of the motor. It demonstrates that the atoms on the adjacent ends between motor and rotor have been bonded and become $s p^{2}$ atoms (see Fig. 2(a)).

For the MC condition, Fig. 3(a) shows that the rotational frequency fluctuates obviously between 75 and $125 \mathrm{GHz}$ (mean value $100 \mathrm{GHz}$ ). The rotational frequency of the inner tube (blue line) is about half the rotational frequency of the motor (yellow broken line). This means that rotations of rotor and motor are asynchronous. The historical positions of MCITs (blue line) shown in Fig. 3(b) also demonstrate that the interaction between motor and rotor tends to be stable after 3500 ps for the present model.

For the BC condition, Fig. 3(a) shows that the rotation of the rotor is very difficult to activate. For example, the rotational frequency of the rotor (red line) varies near zero for the first 4000 ps. We describe that duration as "smooth time." When we extend the simulation time to $10 \mathrm{~ns}$, the rotational frequency of the rotor varies between 25 and $75 \mathrm{GHz}$ after $4000 \mathrm{ps}$. The mean value of the rotational frequency of the rotor is the lowest among the three cases of end configuration.

From the above analysis, we know that the rotational frequency of the rotor is triggered by the interaction between the right end of the motor and the left end of the rotor. From a mechanics point of view, the interaction between rotor and rotating motor creates a torque (axial moment, Table I) on the rotor, and further nonzero angular acceleration of the rotor appears. At the same time, an interaction exists between rotor and outer tube in the bearing. The interlayer friction $^{6,23}$ increases with the increase of rotor's rotational frequency. Hence, the rotational frequency of the rotor has a peak value that is no higher than that of the motor. The obvious fluctuation of torque (standard deviation is far greater than the mean value) is mainly due to thermal vibration of atoms on tubes.

\section{B. Effect of radii difference between motor and rotor}

In the cases considered above, the chirality of the motor is the same as that of the rotor in the bearing. We next investigate the effects of interaction between $s p^{2}$ and/or $s p^{1}$ bonded carbon atoms on rotation transmission. It is recognized that interaction near the joint between motor and rotor is influenced by the orbital hybridization types of atoms. Hence, to design an asynchronous rotation transmission system, we may also achieve the desired results by adjusting the difference between the diameters of motor and rotor. Here, we choose five armchair tubes as the motor to drive the same bearing from a DWCNT of $(5,5) /(10,10)$. In all cases, both motor and rotor have open ends.

TABLE I. Average torque applied on rotor and the standard deviation of torque with different joint configuration.

\begin{tabular}{lccc}
\hline \hline & BO & MC & BC \\
\hline Average torque (eV) & 0.28104 & 0.01185 & 0.00072 \\
Standard deviation $(\mathrm{eV})$ & 4.8379 & 0.41094 & 0.31818 \\
\hline \hline
\end{tabular}

Fig. 4(a) presents the rotational frequency of rotors driven by motors from six different CNTs. In the top layer of Fig. 4(a), the rotor driven by either a $(5,5)$ or a $(6,6)$ motor shows very stable synchronous rotation. The mid-layer of Fig. 4(a) shows the histories of rotational frequency of the rotor driven by either a $(7,7)$ or an $(8,8)$ motor. Here too, both average values of rotational frequency of the rotor are very close to that of motor. But the other curves in the bottom layer of Fig. 4(a) show that the rotational frequency of the rotor is not identical to that of the motor, i.e., rotation transmission is asynchronous. For example, the rotational frequency of the rotor driven by a $(9,9)$ motor is only $170 \mathrm{GHz}(<250 \mathrm{GHz}$ of the motor). It is similar to the system with hydrogen atoms bonded at the joint between $(9,9)$ motor and rotor in our recent work. ${ }^{28}$ The transmission effect for the system with such radius difference between motor and rotor is mainly caused by the interlayer van der Waals forces rather than the pattern of hybridization at the tube end. When the van der Waals force performs dominantly during rotational transmission, the asynchronous rotational frequency can be generated. Similarly, for the $(10,10)$ motor, which has the same chirality as the outer tube in the
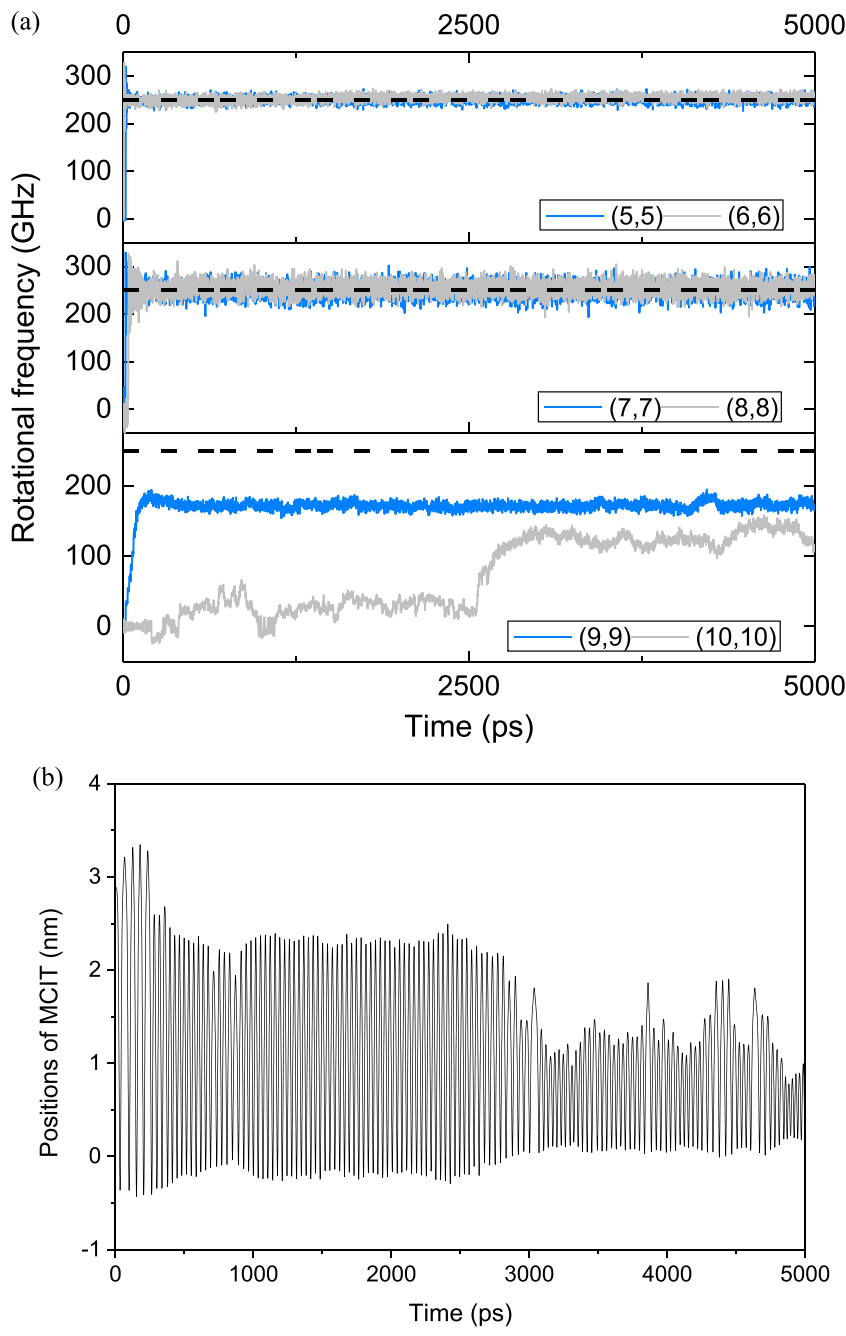

FIG. 4. Histories of (a) rotational frequency for different chirality of motor (broken lines are the rotational frequency of motor) and (b) positions of MCIT driven by $(10,10)$ motor. 
DWCNT bearing, the rotation of the rotor is unstable, with the highest value of rotational frequency of $<160 \mathrm{GHz}$. At the same time, the whole system acts as an oscillator, as studied by Kang et al. ${ }^{32}$ with the translational motion of the rotor along axis (Fig. 4(b)).

Figs. 5(a) and 5(b) show the configurations of a system with a $(7,7)$ motor at 1810 ps and 1836 ps, separately. Because a strong interaction occurs at the joint between motor and rotor, the right end of the motor has an asymmetric deformation. Moreover, a similar deformation appears for the $(8,8)$ rotor. Such an asymmetric end leads to eccentric rotation of the rotor. Correspondingly, the outer tube also has obvious deformation (Figs. 5(a) and 5(b)). From Figs. 5(c)-5(e), the radii differences between motor and rotor are large enough so that they cannot form new $s p^{2}$ atoms. Thus, we may conclude that such a system is not suitable for stable rotation transmission when the difference between radii of motor (i.e., $(7,7)$ and $(8,8)$ ) and rotor (i.e., $(5,5))$ is in the range of $(0.13,0.21) \mathrm{nm}$. If the radii difference between motor and rotor is large than $0.275 \mathrm{~nm}$ (i.e., $(9,9)$ motor and $(5,5)$ rotor), the asynchronous transmission results can be generated due to van der Waals interaction.

\section{Effect of motor' rotational frequency}

Two representative motors are discussed, e.g., $(9,9)$ and $(10,10)$ motor. For $(9,9)$ motor, it can excite stable asynchronous rotation of rotor. For $(10,10)$ motor, it will drive rotor acting as an oscillator, simultaneously. (a)

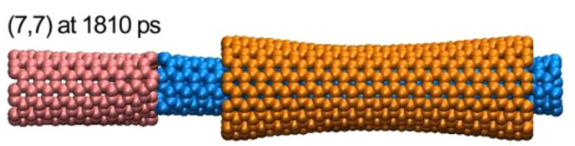

(b)

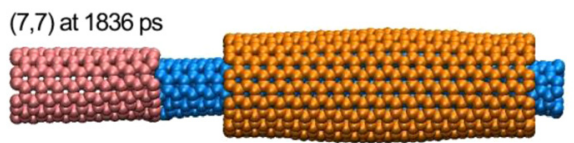

(c)

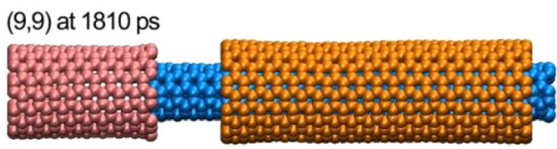

(d)

$(10,10)$ at $1794 \mathrm{ps}$

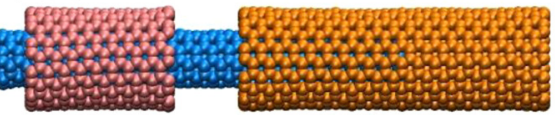

(e)
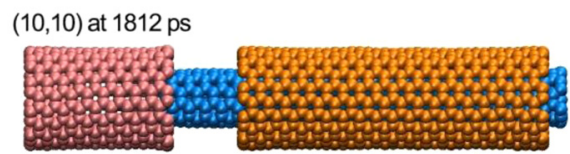
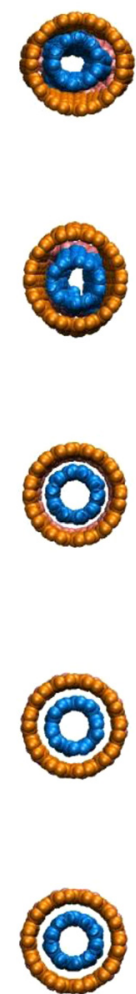

FIG. 5. Snapshots of simulation models (a) with $(7,7)$ motor at $1810 \mathrm{ps}$ and (b) $1836 \mathrm{ps}$, (c) with $(9,9)$ motor at $1810 \mathrm{ps}$, (d) with $(10,10)$ motor at 1794 ps and (e) 1812 ps.

\section{Driven by $(9,9)$ motor}

Here, we just show the results of the rotor driven by super high-speed $(9,9)$ motor (Fig. 6). As the rotational frequency of motor is over $230 \mathrm{GHz}$, the rotational frequency of rotor increases approximately linear with the rotational frequency of motor. The standard deviation of rotational frequency of rotor is about $5 \mathrm{GHz}$. It is significant to give a design of a RTS with stable and adjustable rotating state of rotor.

\section{Driven by $(10,10)$ motor}

In the pre-pullout oscillation work, ${ }^{2,15}$ the inner tube amplitude damps with time continuously. In our previous work, ${ }^{12}$ if the rotor has a high rotational speed, it will oscillate in the stator, simultaneously. Fig. 7 demonstrates both of the oscillation and rotation of the rotor when driven by the $(10,10)$ motor. In the system, both the motor and stator are made from the $(10,10)$ CNT. From Kang et al. ${ }^{32}$ the oscillation of the inner tube depends on the distance between the two outer tubes. From Fig. 7(a), the stable oscillation of the rotor happens during $[3000,5000]$ ps when driven by $240 \mathrm{GHz}$ motor or during [1000, 2500] ps when driven by $250 \mathrm{GHz}$ motor. The stable oscillation frequency is around $47 \mathrm{GHz}$, that can match up with the fitting result by Kang et $a l .{ }^{32}$ During the same period, the rotation of the rotor is stable, too. For example, the average values of the rotational frequency of the rotor are $\sim 144$ and $\sim 27 \mathrm{GHz}$, respectively (Fig. 7(b)). This phenomenon demonstrates that the highspeed rotating inner tube will result in a stable oscillation of the inner tube in the outer tube(s) with higher radius. ${ }^{12}$ When the rotational frequency is 230,260 , or $270 \mathrm{GHz}$, the oscillation fluctuates obviously. For such system, the activated translational and rotational motion of rotor can be regarded as multi-signal transmission in NEMS.

\section{Effect of temperature}

Here, only super high-speed rotational transmission is under consideration, i.e., the rational frequency of motor is $250 \mathrm{GHz}$. Four temperature cases are considered, i.e., 150, 300,500 , and $800 \mathrm{~K}$.

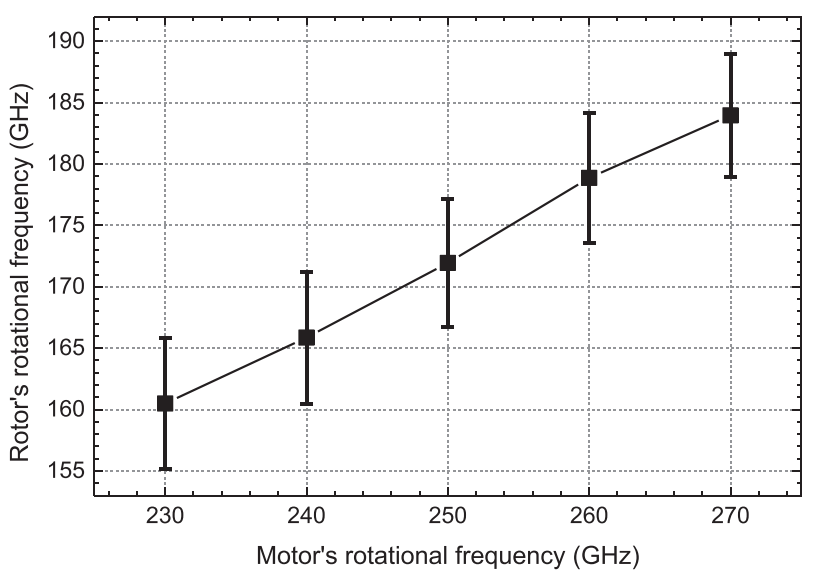

FIG. 6. The rotational frequency of rotor vs. $(9,9)$ motor with super high rotational speed after 1000 th ps. 
(a) Oscillation of rotor

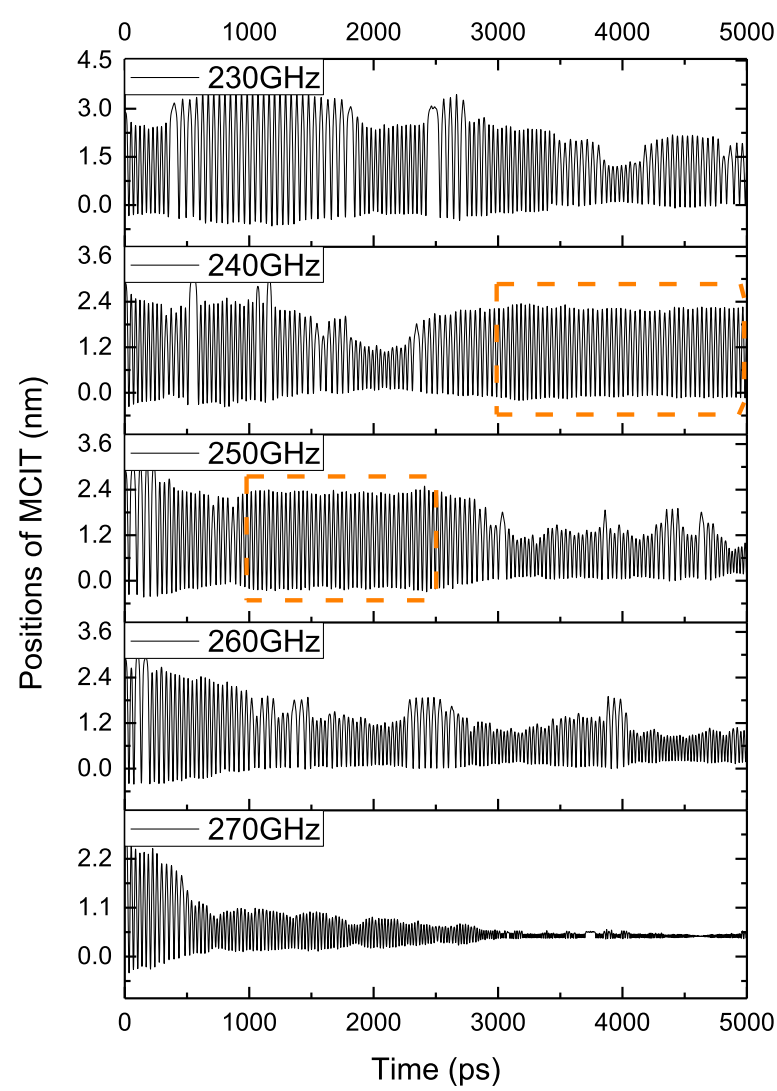

(b) Rotation of rotor

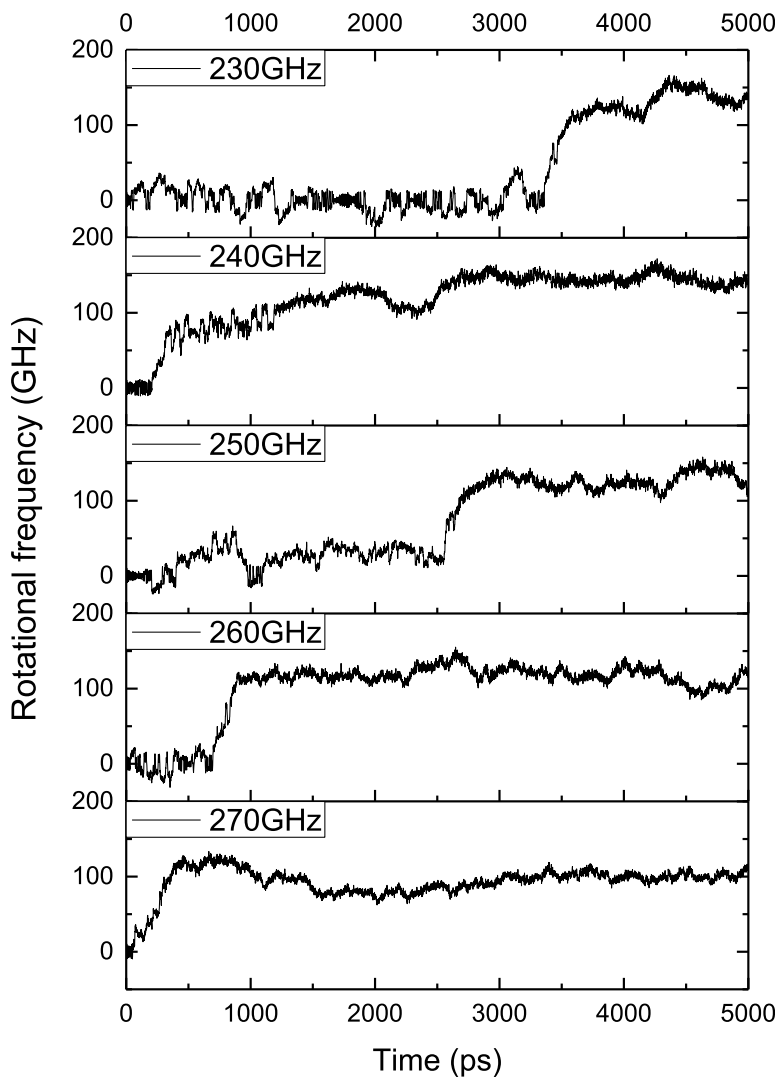

FIG. 7. The rotational frequency of rotor vs. $(10,10)$ motor's super high rotational frequency.

Fig. 8(a) lists the rotational frequency of rotor driven by $(9,9)$ motor at different environmental temperature. It is found that the rotor rotates synchronously with motor at 150 , 500 , and $800 \mathrm{~K}$. Only when the system is at $300 \mathrm{~K}$, the nonsynchronous rotational transmission happens.

In Fig. 8(b), the rotational transmission histories are listed when the rotor is driven by $(10,10)$ motor. The results show that the environmental temperature influences the rotational transmission more obviously. When the system is at $150 \mathrm{~K}$, the synchronous rotational transmission happens. As observing the configuration of the motor-rotor joint, we find that the motor and rotor are attracted to each other tightly and the motor is under buckling. So, we concluded that the synchronous transmission happens easily at lower environmental temperature. Although motor and rotor are attracted each other and synchronous rotational transmission happens at $800 \mathrm{~K}$ for both of $(9,9)$ and $(10,10)$ motors, the mechanism is different from that at $150 \mathrm{~K}$. From the configuration of the motor-rotor joint, we find that the end of motor is not under buckling. It is actually because of higher phonon vibration on motor which leads to the easier happening of bonding between atoms on motor and rotor.

\section{CONCLUSIONS}

In summary, we investigate a series of rotational transmission systems made by coaxial CNTs with MD simulation. The results indicate that the interaction between the motor and rotor is influenced by such factors as the bonding types of carbon atoms near their joint, the difference between their radii, rotational frequency of motor, and environmental temperature. Following concluding remarks can be drawn.

First, if the joint between motor and/or rotor has pure $s p^{2}$ bonded atoms (BC, MC), the interaction is not strong enough to drive the rotor rotating synchronously. The $\mathrm{MC}$ condition can generate higher rotational frequency than $\mathrm{BC}$ condition because of the stronger effect of the $s p^{1}$ bonded atoms. All the synchronous rotation of rotor is due to the original $s p^{1}$ atoms on the motor and rotor becoming new $s p^{2}$ atoms.

Second, the radii difference between motor and rotor influences the transmission behavior, too. To design a stable synchronous RTS, the radii difference between motor and rotor should be no more than $0.13 \mathrm{~nm}$. If the radii difference is over $0.275 \mathrm{~nm}$, van der Waals interaction between tube walls can drive an asynchronous rotation of rotor.

Third, when the motor's rotational frequency is between $230 \mathrm{GHz}$ and $270 \mathrm{GHz}$, a stable asynchronous transmission result can be observed and the rotational speed of rotor almost increases linearly with the increase of the rotational speed of $(9,9)$ motor. When driven by the $(10,10)$ motor, the system may be considered as a multi-signal transmission device due to the coexistence of the oscillation and rotation of the rotor.

Finally, environmental temperature also has an obvious influence on the effects of rotational transmission of the 
(a) Driven by $(9,9)$ motor

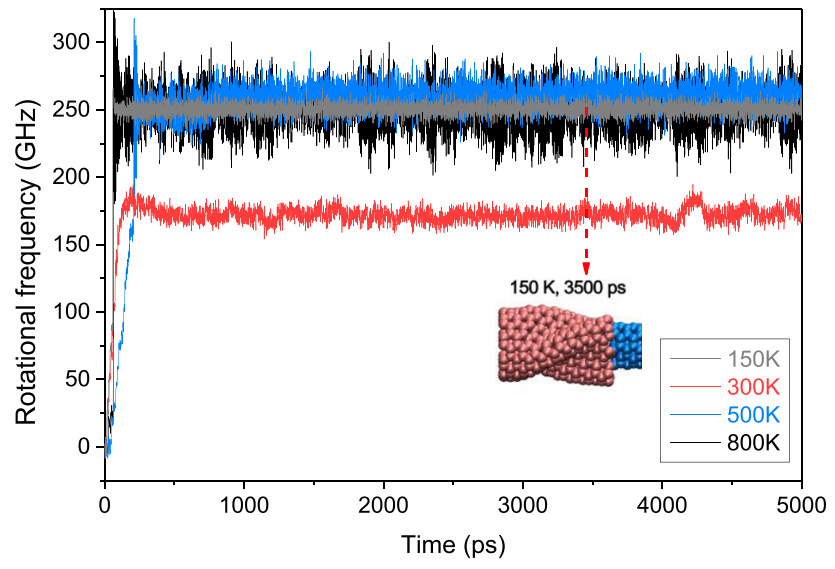

(b) Driven by $(10,10)$ motor

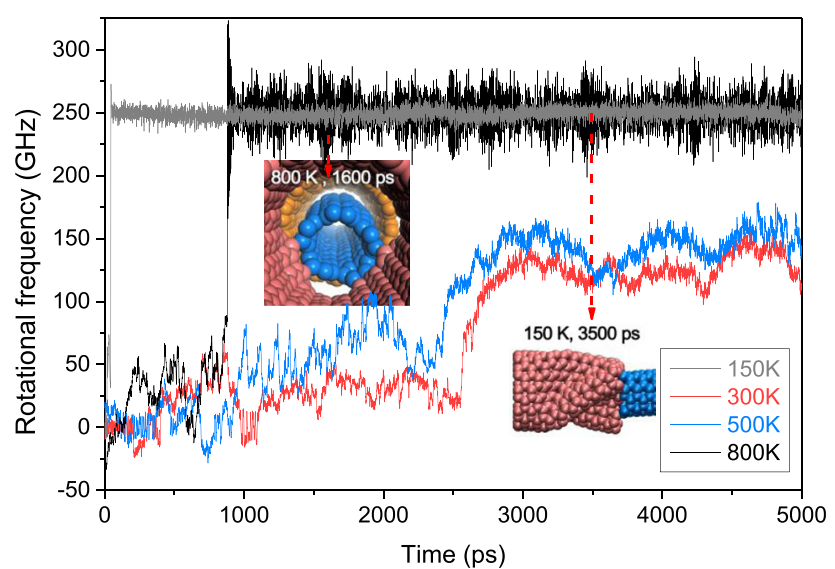

FIG. 8. The rotational transmission histories of rotor driven by $(9,9)$ and $(10,10)$ motors at different temperature.

system. When the environmental temperature of is high and/ or the difference between motor and rotor radii is low, the new $s p^{2}$ atoms appear easily. When the environmental temperature is low (e.g., <150), the open end of motor is easily under buckling due to the attraction of the rotor. The appearing of some new $s p^{2}$ bonded atoms on the adjacent ends of motor and rotor leads to their synchronous rotation.

\section{ACKNOWLEDGMENTS}

Financial support from the National Natural-ScienceFoundation of China (Grant Nos. 50908190 and 11372100) is acknowledged.
${ }^{1}$ H. Somada, K. Hirahara, S. Akita, and Y. Nakayama, Nano Lett. 9, 62 (2008)

${ }^{2}$ W. Guo, Y. Guo, H. Gao, Q. Zheng, and W. Zhong, Phys. Rev. Lett. 91, 125501 (2003).

${ }^{3}$ P. A. Schoen, J. H. Walther, S. Arcidiacono, D. Poulikakos, and P. Koumoutsakos, Nano Lett. 6, 1910 (2006).

${ }^{4}$ W. Qiu, Y.-L. Kang, Z.-K. Lei, Q.-H. Qin, Q. Li, and Q. Wang, J. Raman Spectrosc. 41, 1216 (2010).

${ }^{5}$ Z. Qin, Q.-H. Qin, and X.-Q. Feng, Phys. Lett. A 372, 6661 (2008).

${ }^{6}$ Q. W. Hou, B. Y. Cao, and Z. Y. Guo, Nanotechnology 20, 495503 (2009).

${ }^{7}$ J. Servantie and P. Gaspard, Phys. Rev. Lett. 97, 186106 (2006).

${ }^{8}$ J. Cumings and A. Zettl, Science 289, 602 (2000).

${ }^{9}$ M.-F. Yu, B. I. Yakobson, and R. S. Ruoff, J. Phys. Chem. B 104, 8764 (2000).

${ }^{10}$ B. Bourlon, D. C. Glattli, C. Miko, L. Forró, and A. Bachtold, Nano Lett. 4, 709 (2004).

${ }^{11}$ A. Fennimore, T. Yuzvinsky, W.-Q. Han, M. Fuhrer, J. Cumings, and A. Zettl, Nature 424, 408 (2003).

${ }^{12}$ K. Cai, H. Yin, Q. Qin, and Y. Li, Nano Lett. 14, 2558 (2014).

${ }^{13}$ S. B. Legoas, V. R. Coluci, S. F. Braga, P. Z. Coura, S. O. Dantas, and D. S. Galvão, Phys. Rev. Lett. 90, 055504 (2003).

${ }^{14}$ Q. Zheng and Q. Jiang, Phys. Rev. Lett. 88, 045503 (2002).

${ }^{15}$ J. L. Rivera, C. McCabe, and P. T. Cummings, Nanotechnology 16, 186 (2005).

${ }^{16}$ M. Hamdi, A. Subramanian, L. Dong, A. Ferreira, and B. J. Nelson, IEEE/ ASME Trans. Mechatron. 18, 130 (2013).

${ }^{17}$ I. Santamaría-Holek, D. Reguera, and J. M. Rubi, J. Phys. Chem. C 117, 3109 (2013).

${ }^{18}$ Z. Tu and X. Hu, Phys. Rev. B 72, 033404 (2005).

${ }^{19}$ A. Subramanian, L. X. Dong, J. Tharian, U. Sennhauser, and B. J. Nelson, Nanotechnology 18, 075703 (2007).

${ }^{20}$ R. Saito, R. Matsuo, T. Kimura, G. Dresselhaus, and M. Dresselhaus, Chem. Phys. Lett. 348, 187 (2001).

${ }^{21}$ Y. E. Lozovik, A. V. Minogin, and A. M. Popov, Phys. Lett. A 313, 112 (2003).

${ }^{22}$ A. V. Belikov, Y. E. Lozovik, A. G. Nikolaev, and A. M. Popov, Chem. Phys. Lett. 385, 72 (2004).

${ }^{23}$ A. Barreiro, R. Rurali, E. R. Hernandez, J. Moser, T. Pichler, L. Forro, and A. Bachtold, Science 320, 775 (2008).

${ }^{24}$ Z. Guo, T. Chang, X. Guo, and H. Gao, Phys. Rev. Lett. 107, 105502 (2011).

${ }^{25}$ J. W. Kang and H. J. Hwang, Nanotechnology 15, 1633 (2004).

${ }^{26}$ J. Zhao, J.-W. Jiang, L. Wang, W. Guo, and T. Rabczuk, J. Mech. Phys. Solids 71, 197 (2014).

${ }^{27}$ Q. Wang and B. Arash, Comput. Mater. Sci. 82, 350 (2014).

${ }^{28}$ K. Cai, H. Yin, N. Wei, Z. Chen, and J. Shi, Appl. Phys. Lett. 106, 021909 (2015).

${ }^{29}$ LAMMPS Molecular Dynamics Simulator, 2013 (http://lammps.sandia.gov/).

${ }^{30}$ S. J. Stuart, A. B. Tutein, and J. A. Harrison, J. Chem. Phys. 112, 6472 (2000)

${ }^{31}$ P. Giannozzi, S. Baroni, N. Bonini, M. Calandra, R. Car, C. Cavazzoni, D. Ceresoli, G. L. Chiarotti, M. Cococcioni, I. Dabo, A. Dal Corso, S. de Gironcoli, S. Fabris, G. Fratesi, R. Gebauer, U. Gerstmann, C. Gougoussis, A. Kokalj, M. Lazzeri, L. Martin-Samos, N. Marzari, F. Mauri, R. Mazzarello, S. Paolini, A. Pasquarello, L. Paulatto, C. Sbraccia, S. Scandolo, G. Sclauzero, A. P. Seitsonen, A. Smogunov, P. Umari, and R. M. Wentzcovitch, J. Phys.: Condens. Matter 21, 395502 (2009).

${ }^{32}$ J. W. Kang, K.-S. Kim, H. J. Hwang, and O. K. Kwon, Phys. Lett. A 374, 3658 (2010). 